\title{
Quadratus lumborum block for perioperative analgesia in patients treated with abdominoplasty. A randomised controlled trial
}

\author{
T. W. Bjelland, T. G. R. Yates, U. J. Spreng. Dept of Anesthesia, Intensive Care and \\ Emergencies, Bærum Hospital - Sandvika (Norway)
}

\begin{abstract}
Background and Goal of Study: Postbariatric abdominoplasty involves removing large amounts of excess skin and subcutaneous tissue. The quadratus lumborum (QL)-block provides regional analgesia of the abdominal wall. The goal of study was to investigate the effect of a QL-block as part of a multimodal analgesia regimen in postbariatric patients treated with standard full abdominoplasty.
\end{abstract}

Materials and Methods: Randomised, placebo-controlled, triple blind study $(n=50)$. All patients received perioperative paracetamol and peroperative local infiltration anaesthesia. QLblock was administered bilaterally before induction of general anaesthesia with $40 \mathrm{ml}$ of either ropivacaine $0.375 \%(n=25)$ or saline $0.9 \% \quad(n=25)$. Patients received patient controlled iv analgesia (1 mg morphine intravenously with a 5 minute lockouttime). Opioid use the first 24 postoperative hours were converted to morphine equivalent units (MEQ) using a standard table. Pain was reported on the numerical rating scale (NRS) at the postoperative ward, and at 12, 24 and 48 hours postoperatively and converted to area under the curve (AUC) using the linear trapezoid rule. Sample size was estimated to 23 patients in each group.
Results and Discussion: 4 patients were excluded due to reoperation $(n=2)$, and deviation from study protocol $(n=2)$. Patient characteristics were similar between groups. For the primary endpoint, MEQ were similar between groups during the first 24 hours with mean 26 (25) vs 33 (33) mg (Fig 1) $(p=.22$, administered treatment in group $A$ and $B$ censored due to triple blinding). NRS AUC was also similar between groups ( $p=.68$ and .55 during rest and coughing, respectively). Technical challenges in administering the block was reported in 13 of 46 patients and the lack of an opioid-sparing effect suggest the block should be reserved for individual cases in this population.

Conclusion: The QL-block did not reduce opioid use or pain after standard full abdominoplasty in postbariatric patients.

We acknowledge Dr Frøyen, Dr Lysebråten, Elisabet Anderson, all colleagues at our dept, nurses at the postoperative and gynaecological wards, and the nurse Pain-team for invaluable contributions.

\section{Accumulated dose first 24 postoperative hours}

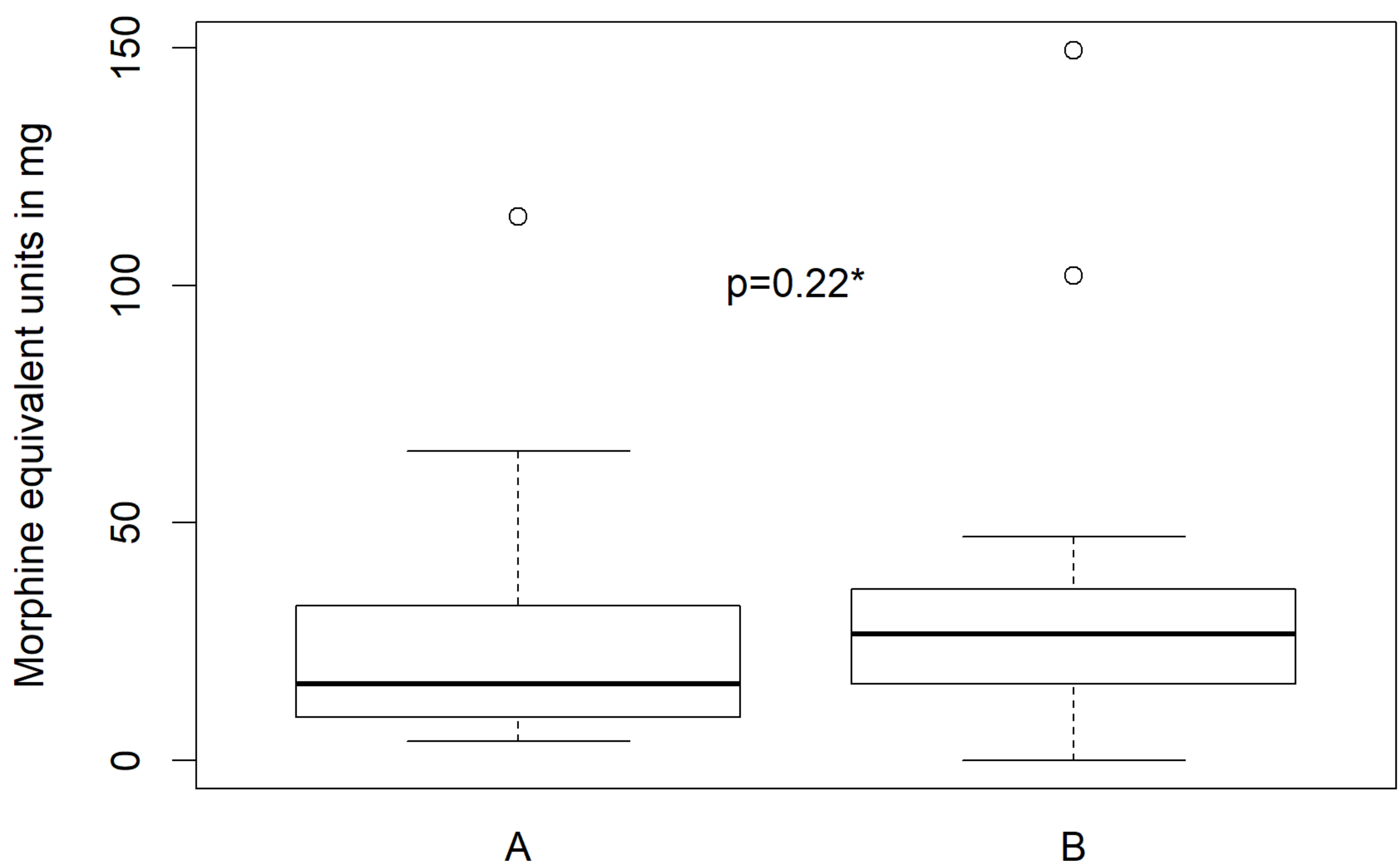

*Morphine equivalent units were log-transformed before one-sided Student's T-test performed 